\title{
Performance Enhancement of MIMO-OFDM using Redundant Residue Number System
}

\author{
M. I. Youssef, A. E. Emam, M. Abd Elghany
}

Department of Electrical Engineering, Faculty of Engineering, Al- Azhar University, Egypt

\begin{tabular}{l} 
Article Info \\
\hline Article history: \\
Received May 24, 2018 \\
Revised Aug 20, 2018 \\
Accepted Aug 27, 2018 \\
\hline
\end{tabular}

Keyword:

Conventional codes

Equalizers

Error detection and correction

Redundant residue number

system;

Wireless communication

\begin{abstract}
Telecommunication industry requires high capacity networks with high data rates which are achieved through utilization of Multiple-Input-MultipleOutput (MIMO) communication along with Orthogonal Frequency Division Multiplexing (OFDM) system. Still, the communication channel suffers from noise, interference or distortion due to hardware design limitations, and channel environment, and to combat these challenges, and achieve enhanced performance; various error control techniques are implemented to enable the receiver to detect any possible received errors and correct it and thus; for a certain transmitted signal power the system would have lower Bit Error Rate (BER). The provided research focuses on Redundant Residue Number System (RRNS) coding as a Forward Error Correction (FEC) scheme that improves the performance of MIMO-OFDM based wireless communications in comparison with current methods as Low-Density Parity Check (LDPC) coders at the transmitter side or equalizers at receiver side. The Bit Error Rate (BER) performance over the system was measured using MATLAB tool for different simulated channel conditions, including the effect of signal amplitude reduction and multipath delay spreading. Simulation results had shown that RRNS coding scheme provides an enhancement in system performance over conventional error detection and correction coding schemes by utilizing the distinct features of Residue Number System (RNS).
\end{abstract}

Copyright $@ 2018$ Institute of Advanced Engineering and Science. All rights reserved.

\section{Corresponding Author:}

M. Abd Elghany,

Department of Electrical Engineering,

Faculty of Engineering,

Al-Azhar University,

Nasr City, Cairo - Egypt.

Email: mohamedgheth@yahoo.com

\section{INTRODUCTION}

Multiple-Input-Multiple-output (MIMO) antenna system along with Orthogonal Frequency Division Multiplexing (OFDM) scheme provide an attractive wireless communication solution for future wireless networks through providing high-data-rate wireless access at high quality of service (QoS) taking into accounts that the spectrum is a rare resource element and propagation conditions are difficult due to existence of fading and interference from other users [1].

MIMO wireless technology from its side provides enhanced spectrum efficiency through spatial multiplexing gain, and improved communication link reliability due to transmit system diversity gain [2]. At the same time OFDM distribute data over multiple numbers of closely spaced orthogonal carriers providing higher spectral efficiency by spacing the channels closer together without fearing from harmful effect of inter-carrier interference as carriers are orthogonal to each other. 
The wireless communication systems are defined in IEEE standards (802.11n WLAN and 802.16 WMAN), based on the combination of MIMO communication system with Orthogonal Frequency Division Multiplexing modulation (MIMO-OFDM), and even On-going next generation communication systems are based on MIMO-OFDM wireless systems.

As wireless digital networks are prone to bit errors during transmission, error detection and correction techniques are implemented to reduce bit-error effects and ensure receiver eventually is able to restore the correct packet of information.

In this paper a proposal for a new coding scheme for error detection and correction using RRNS instead of the current error control codes used, where RRNS shows enhanced system performance over conventional error correction schemes, for MIMO-OFDM based wireless communication system.

The paper starts with a background on the Forward Error Correction techniques as seen in Section 2, then in Section 3 the Residue Number System and redundancy features and applications are given, followed in Section 4 by illustration for RRNS error detection and correction implementation. In Section 5, the overall system architecture is provided, and in Section 6 methods of system evaluation are given. In Section 7 simulation results are provided to analyze the system performance and finally the conclusion is given in Section 8.

\section{FORWARD ERROR CORRECTION TECHNIQUES}

Forward Error Correction (FEC) are methods which are used to enhance the channel capacity through adding redundant data to the message in a way that it can be restored at the receiving side even if there are a errors present during the transmission process. This redundant data allow receiver to detect and correct errors without needing to retransmit the message again and without requiring a handshaking process between Transmit/Receive systems [3].

The FEC scheme provides great advantage in noisy channels where a large number of retransmissions would be required before a packet is received free of errors. It is also used in cases where no feedback exists between the receiver and the transmitter.

The encoded message could be systematic coded if portion of the output is directly resembling the input or non-systematic coded if the output is a modified form of the original information through shuffling original message symbols across several code using an interleaver to improve the performance of FEC codes, and thus the errors would have a more uniform distribution form [4].

The coding technique used in FEC schemes could be categorized to Block Error correction codes, and Convolutional Error correction codes. The first ones are as Hamming, BCH, Reed-Solomon, and turbo codes, while the second ones are as Viterbi, and Low Density Parity Check Code [5], [6].

\section{RESIDUE NUMBER SYSTEM REVIEW}

The RNS provides a representation of large integers through set of smaller ones, such that arithmetic computation performed in an efficient matter.

The Residue numbers has unique features, as it is a carry-free arithmetic, which implies its ability to perform the operations related to the individual residue digits of different moduli independently. As well as the residue representations carry no weight-information and hence an error in any digit-position in a given representation does not affect other digit-positions [7].

The RNS is defined through selecting $v$ positive pair-wise relative primes $\mathrm{m}_{\mathrm{i}}(\mathrm{i}=1,2,3 \ldots v)$ referred to as moduli, such that any integer $\mathrm{N}$, describing a message, is given by the sequence $\left(\mathrm{r}_{1}, \mathrm{r}_{2} \ldots \mathrm{r}_{v}\right)$ in the range $0<\mathrm{N}<\mathrm{M}_{\mathrm{I}}$ in a unique matter, where;

$\mathrm{r}_{\mathrm{i}}=\mathrm{N}\left(\bmod \mathrm{m}_{\mathrm{i}}\right)$; The residue digit of $\mathrm{N}$ upon division by $\mathrm{m}_{\mathrm{i}}$

Where;

$r_{i}$ is least positive remainder when $\mathrm{N}$ is divided by modulus $\mathrm{m}_{\mathrm{i}}$

$\mathrm{M}_{\mathrm{I}}=\prod \mathrm{m}_{\mathrm{i}}$; is the information symbols' dynamic range

Then; to be able to recover symbols, two approaches are available; either through the Chinese Reminder Theorem (CRT) which is a parallel implementation scheme or Mixed Radix Conversion (MRC) algorithm that is an inherently sequential approach. In the coming subsection a description of both methods are provided. 


\subsection{Chinese remainder theorem method}

The method relies on a mathematical idea that was given in the $4^{\text {th }}$ century AD in china [8]-[10]; where for any given $v$-tuple $\left(\mathrm{r}_{1}, \mathrm{r}_{2} . . \mathrm{r}_{v}\right)$ such that $0 \leq \mathrm{r}_{\mathrm{i}}<\mathrm{m}_{\mathrm{i}}$; there exists one and only one integer $\mathrm{N}$ such that $0 \leq \mathrm{N}<\mathrm{M}_{\mathrm{i}}$ and $\mathrm{r}_{\mathrm{i}}=\mathrm{N}\left(\bmod \mathrm{m}_{\mathrm{i}}\right)$ that allow us to recover the message. The numerical value of $\mathrm{N}$ can be computed according to the equation:

$$
N=\sum r_{i} T_{i} M_{i}(\bmod M) ; \text { for } i: 1 \text { to } n
$$

Where;

$$
\begin{aligned}
& \mathrm{i}=1 \text { to } v ; \\
& \mathrm{M}_{\mathrm{i}}=\mathrm{M} / \mathrm{m}_{\mathrm{i}} ; \mathrm{M} \text { is dynamic range } \\
& \mathrm{T}_{\mathrm{i}}=\mathrm{M}_{\mathrm{i}}^{-1}=\text { inverse value of } \mathrm{M}_{\mathrm{i}} ;
\end{aligned}
$$

Where;

$$
\left|\mathrm{M}_{\mathrm{i}} \times \mathrm{M}_{\mathrm{i}}^{-1}\right|_{\mathrm{mi}}=1
$$

\subsection{Mixed radix conversion method}

For a given set of pair-wise relatively prime moduli $\left\{\mathrm{m}_{1}, \mathrm{~m}_{2}, \ldots, \mathrm{m}_{\mathrm{n}}\right\}$ and a residue state $\left\{\mathrm{r}_{1}, \mathrm{r}_{2} \ldots \mathrm{r}_{\mathrm{n}}\right\}$ of a number $\mathrm{X}$, that number can be uniquely represented in mixed-radix form as seen in next [11]:

$$
\mathrm{X}=\left\{\mathrm{z}_{1}, \mathrm{z}_{2}, \ldots, \mathrm{z}_{\mathrm{n}}\right\}
$$

And;

$$
\mathrm{X}=\mathrm{z}_{1}+\mathrm{z}_{2} \mathrm{~m}_{1}+\mathrm{z}_{3} \mathrm{~m}_{2} \mathrm{~m}_{1}+\ldots . .+\mathrm{z}_{\mathrm{n}} \mathrm{m}_{\mathrm{n}-1} \mathrm{~m}_{\mathrm{n}-2} \ldots . \mathrm{m}_{1} ; 0 \leq \mathrm{z}_{\mathrm{i}} \leq \mathrm{r}_{\mathrm{i}}
$$

So, all what is required is to obtain the value of $z_{i}$ to determine $X$. Where each value of $\mathrm{z}$ is represented as function of the moduli and residue representations as seen in Table 1;

Table 1. Representation of $z_{i}$

\begin{tabular}{cl}
\hline Parameter & Representation \\
\hline $\mathrm{z}_{1}$ & $=\mathrm{r}_{1}$ \\
$\mathrm{z}_{2}$ & $=\|\left.\left.\mathrm{m}_{1}^{-1}\right|_{\mathrm{m} 2}\left(\mathrm{r}_{2}-\mathrm{z}_{1}\right)\right|_{\mathrm{m} 2}$ \\
$\mathrm{z}_{3}$ & $=\|\left.\left(\mathrm{m}_{2} \mathrm{~m}_{1}\right)^{-1}\right|_{\mathrm{m} 3}\left(\mathrm{r}_{3}-\left.\left(\mathrm{z}_{2} \mathrm{~m}_{1}+\mathrm{z}_{1}\right)\right|_{\mathrm{m} 3}\right.$ \\
$\mathrm{z}_{\mathrm{n}}$ & $=\|\left.\left.\left(\mathrm{m}_{\mathrm{n} \ldots \ldots} \ldots \mathrm{m}_{2} \mathrm{~m}_{1}\right)^{-1}\right|_{\mathrm{mn}}\left(\mathrm{r}_{\mathrm{n}}-\mathrm{r}_{\mathrm{n}-1} \mathrm{~m}_{\mathrm{n}-2} \ldots \ldots \mathrm{z}_{2} \mathrm{~m}_{1}+\mathrm{z}_{1}\right)\right|_{\mathrm{mn}}$ \\
\hline
\end{tabular}

And as seen in Table 1 the MRC is considered a sequential process, where obtaining $\mathrm{Z}_{\mathrm{i}}$ requires generating $\mathrm{z}_{\mathrm{i}-1}$ first.

\section{PROPOSED ERROR DETECTION AND CORRECTION ALGORITHM}

Error detection and correction scheme in this paper is proposed using set of RNS moduli as information symbols and additional RNS moduli as redundancy symbols, which is addressed as Redundant Residue Number System (RRNS).

In this scheme each redundant moduli is selected to be greater than any of the other chosen moduli set and don't play any role in determining the system dynamic range. So, an RRNS is obtained by appending an additional $(u-v)$ number of moduli $m_{v+1} ; m_{v+2} ; \ldots . ; m_{u}$, where $m_{v+j} \geq \max \left\{m_{1} ; m_{2} ; \ldots \ldots ; m_{v}\right\}$ is referred to as a redundant modulus, to the previously introduced RNS, in order to form an RRNS of $u$ positive, pairwise relative prime moduli [12], [13].

Now an integer $\mathrm{N}$ in the range $\left[0 ; \mathrm{M}_{\mathrm{I}}\right]$ is represented as a $\mathrm{u}$-tuple residue sequence, $\left(\mathrm{r}_{1} ; \mathrm{r}_{2} ; \ldots \ldots ; \mathrm{r}_{\mathrm{u}}\right)$ with respect to the $u$ moduli. The properties of the RNS indicated in section 3 and specially the property of independence of digits allow recovering the integer $\mathrm{N}$ by any $v$ out of $u$ residue digits using their related moduli, and thus enable the redundant residue number to be used for self-checking, error-detection and 
correction in digital processors as seen in Figure 1. Furthermore, the RRNS approach is the only one that is capable of using the same arithmetic module for generating both the original information part and the parity part of a RRNS codeword [7].

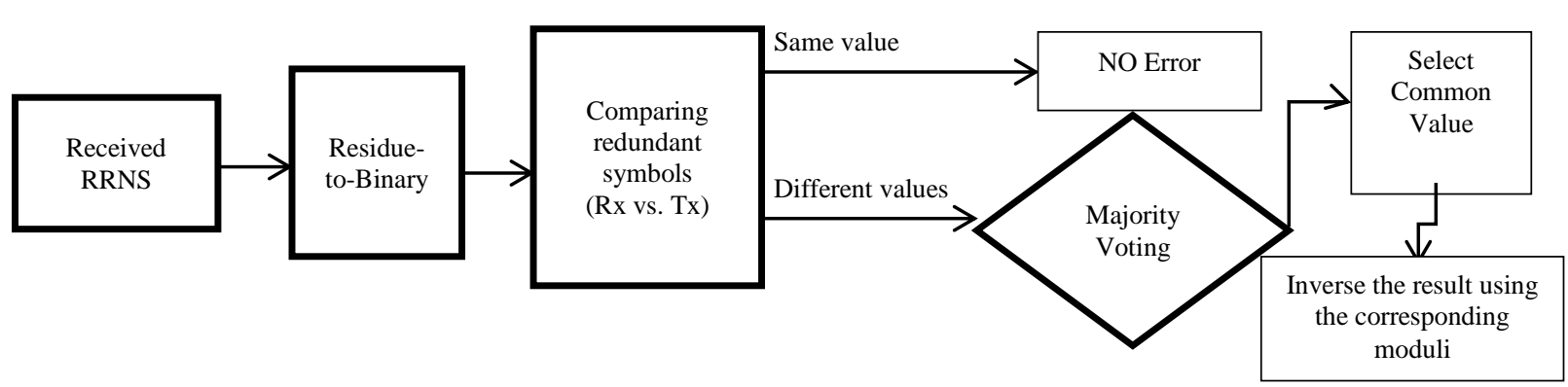

Figure 1. Principle of error detection and correction

From Figure 1 seen above; the received redundant symbols are converted back to binary system and compared to the expected ones, and through this comparison the system would detect the occurrence of an error in the transmitted message.

Then for the correction of the error, the modulus that generated the error must be identified either in $\mathrm{m}_{3}$, or $\mathrm{m}_{2}$ or $\mathrm{m}_{1}$. Using the MRC method [11], a test on each of the information moduli with the two redundant moduli is performed and through this test we are able to identify and correct the bit which generated the error [14]. Thus, through the detection and correction algorithm, the error would be detected and corrected without the need to re-transmit again the information.

\section{SYSTEM MODEL}

The communication system, as shown in Figure 2 is initialized with a binary data random source, which is converted to residue system and protected from errors by adding parity residue symbols using the RRNS encoding algorithm instead of the DVB-S2 LDPC encoder, then the packet is modulated, coded through the Space-Time Block Coding (STBC) encoder, passed to a Serial-To-Parallel (S/P) converter for parallel transmission and then passed through an IFFT block then to the transmission antenna.

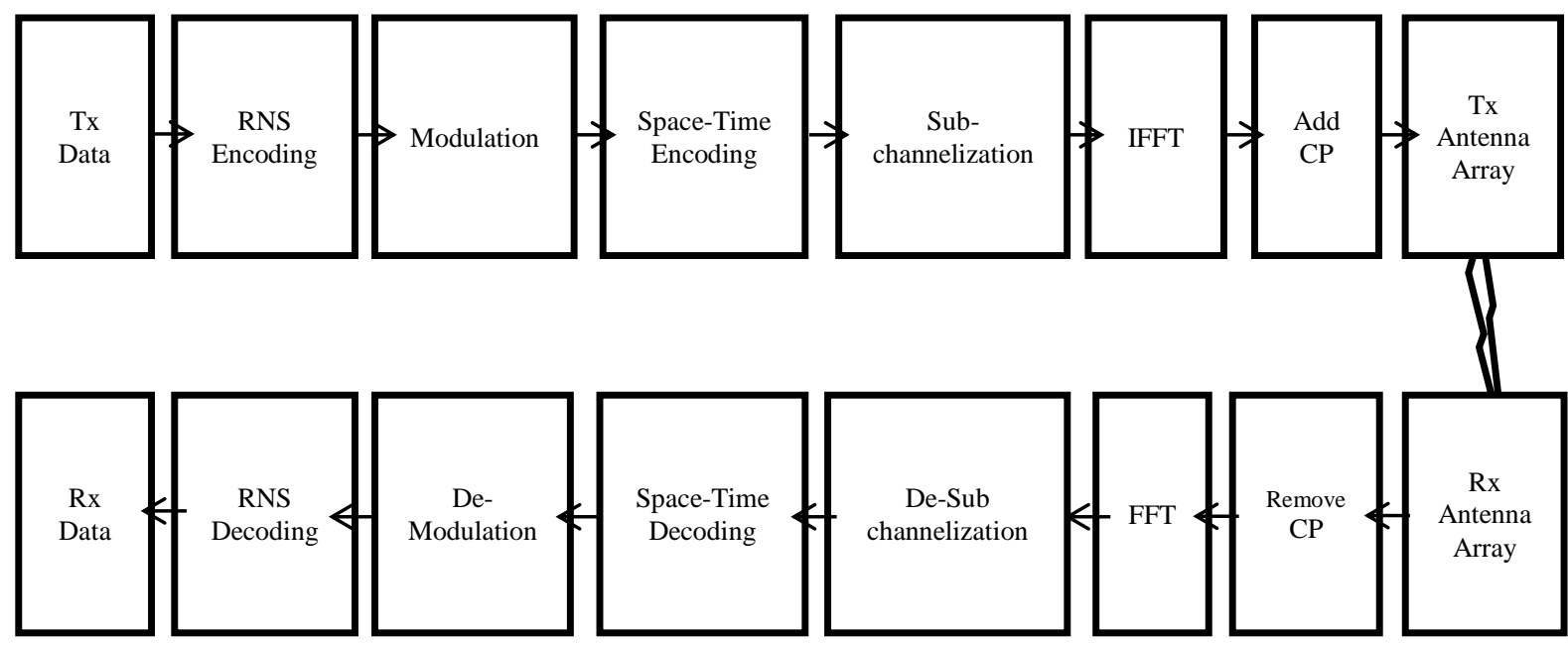

Figure 2. MIMO-OFDM system model

Before transmission a Cyclic prefix (CP) is added to decrease the effect of Inter Symbol Interference (ISI) and Inter Carrier Interference (ICI) caused by the multipath channel [15]. This CP is a copy of the last section of the OFDM symbol that is attached to the front of transmitted OFDM symbol.

To represent the satellite channel in the model presented in Figure 2, several channel fading are given; starting with Additive White Gaussian Noise (AWGN) channel, and then adding multipath fading 
factors through using Rice-Log-Normal distribution (RLN) distribution fading model [16]. The receiver blocks are the reverse blocks of the transmitter.

\section{EVALUATION METHODS}

The performance of the RRNS-based MIMO-OFDM system is evaluated through the parameters defined and presented in this section.

\subsection{Bit error rate (BER)}

The probability of error for MPSK modulated transmission in AWGN is given by:

$$
\begin{aligned}
& \mathrm{P}_{\mathrm{ERR}}=\gamma \sum_{k=1}^{\min \left(2,\left[\frac{M}{4}\right]\right)} Q\left(\sqrt{2 \sigma x} \sin \left(\frac{(2 k-1) \pi}{M}\right)\right) \\
& \gamma=\frac{2}{\max (\log 2 M, 2)}
\end{aligned}
$$

Where;

$M$ is the constellation size, $\rho$ is the SNR per symbol

$\mathrm{x}$ is a chi-square distributed random variable

\subsection{PAPR evaluation method}

The performance is evaluated by measuring the Peak-to-Average Power Ratio (PAPR) of the signal $x(t)$ [17], as seen in Equation (9);

$$
\mathrm{PAPR}=10 \log \left(\max \left|\mathrm{x}_{\mathrm{k}}\right|^{2} / \mathrm{E}\left[\left|\mathrm{x}_{\mathrm{k}}\right|^{2}\right]\right) \text {, in } \mathrm{dB} .
$$

Where; $\mathrm{E}=$ Expectation operator

\subsection{Channel capacity}

Another way to characterize the performance of MIMO channel is the Shannon channel capacity metric. Shannon in [18] defined capacity as the maximum data rate a channel can support at an arbitrarily low error probability. The capacity of MIMO system is given in Equation (10) as;

$$
\mathrm{C}=\log _{2}\left[\operatorname{det}\left(\mathrm{I}_{\mathrm{n}}+\frac{\rho}{N} \mathbf{H} \mathbf{H}^{H}\right)\right]
$$

Where;
$\mathbf{I}_{n}: n \times n$ identity matrix
$N$ : The number of transmit antennas
$\mathbf{H}^{H}$ : Conjugate transpose of $\mathbf{H}$

\section{SIMULATION RESULTS}

The proposed system performance was investigated using MATLAB tool, which involves the transmission of data streams through a FEC coding scheme whose integrity depends on OFDM with 512-ary QAM and Cyclic Prefix (CP):1/8, over different fading channels.

The examination is focused the utilization of either equalizers or coding schemes as an error correction techniques, and analyzing the system performance of such techniques with that using RNS with redundant moduli's as an error detection and correction algorithm.

The MIMO-OFDM system performance is studied through measuring the BER and PAPR over AWGN, Rayleigh and Rician Lognormal (RLN) fading channel conditions.

\subsection{Using equalizers as error correction scheme}

The BER performance as seen in Figure 3, takes into account the simulation of several types of equalizers that are utilized for error correction, which include linear equalizer, a Decision Feedback Equalizer (DFE), and Maximum Likelihood Sequence Estimation (MLSE) equalizer. 


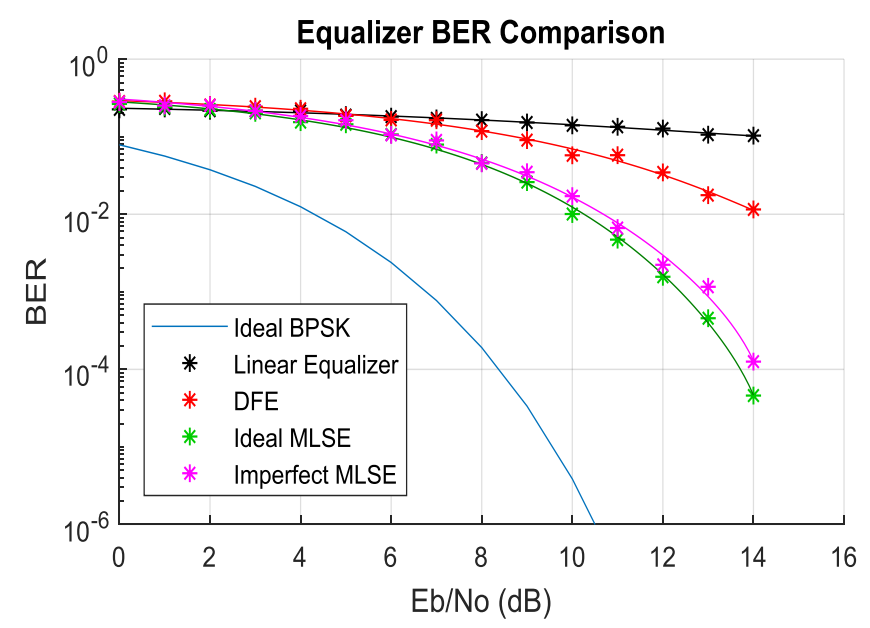

Figure 3. BER vs. $\mathrm{E}_{\mathrm{b}} / \mathrm{N}_{\mathrm{o}}$ for different Equalizer techniques

From Figure 3, we could see the enhanced performance of MLSE over DFE and linear equalizer, and thus we focus on analyzing the performance of MIMO-OFDM with RNS coding system with and without MLSE equalizer over the RLN + AWGN channel, as seen in Figure 4.

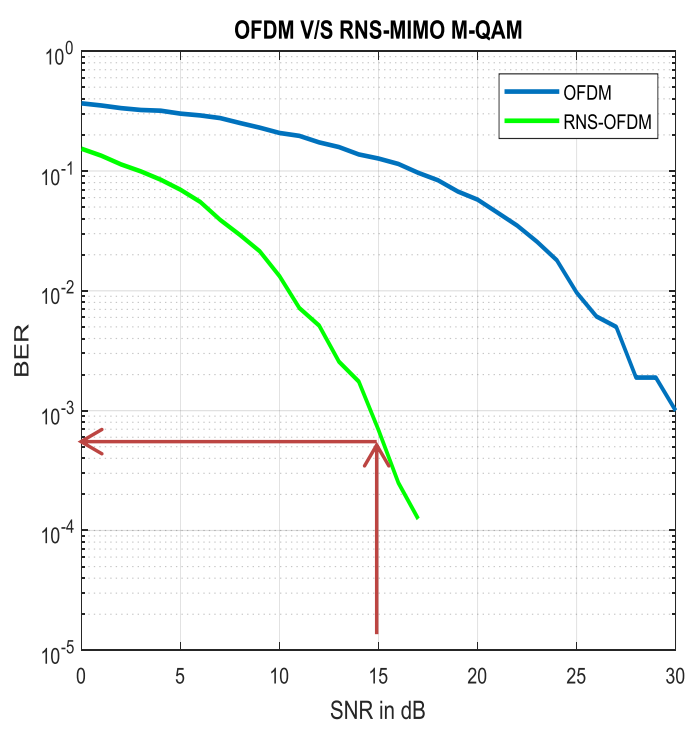

With Equalization

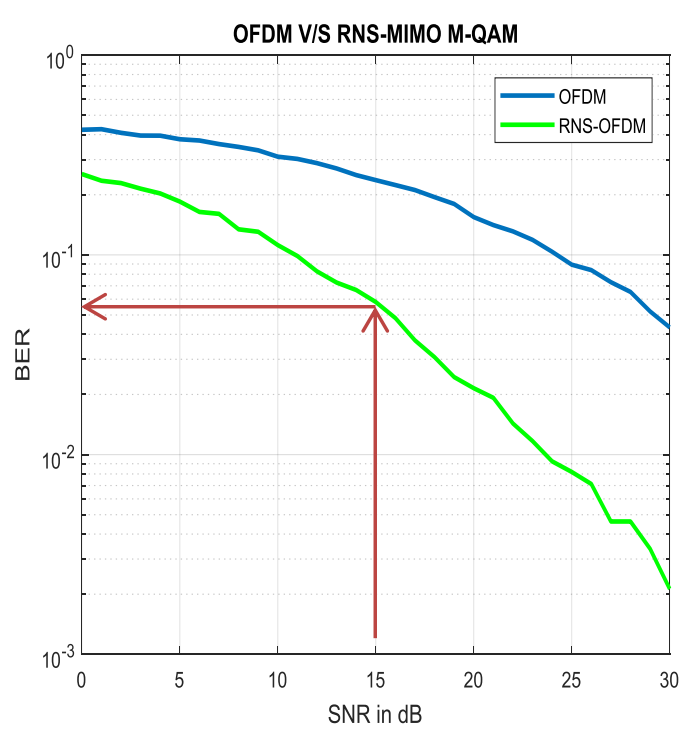

Without Equalization

Figure 4. MIMO-OFDM RNS system with/without Equalizer

From Figure 4, we could notice that at $\mathrm{SNR}=15$, the BER for the communication system with error correction is $1 * 10^{-3}$ while it reaches $5^{*} 10^{-2}$ for the system without error correction.

\subsection{BER performance with current coding correction schemes}

The performance over AWGN channels for current error correction schemes that utilize coding approach as seen in Figure 5 shows that Golay code (which is a Block Error correction type) provides the best error correction code compared to other cods.

The current generation of linear block codes uses LDPC coders which utilize high multiplexing capacity, and are differentiated from Golay codes through the way they are decoded. So, while binary Golay codes are decoded through algebraic methods, LDPC codes are iteratively decoded. Thus, in the next subsections analysis will be focused on LDPC coders. 


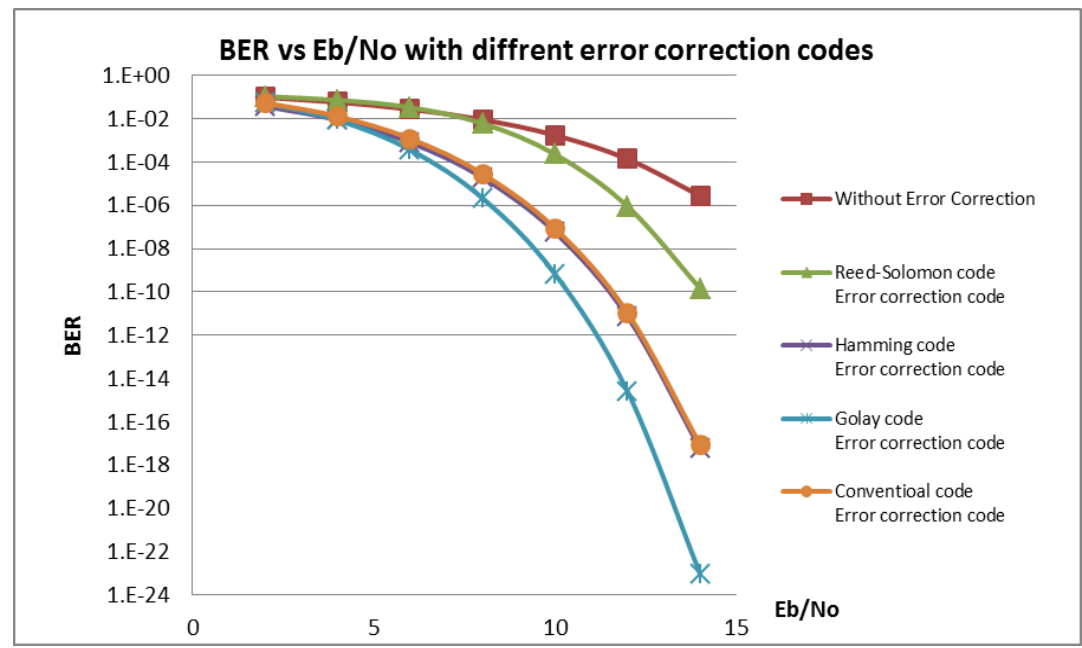

Figure 5. BER vs. $\mathrm{E}_{\mathrm{b}} / \mathrm{N}_{\mathrm{o}}$ for current correction codes

\subsection{Using coding techniques as error correction scheme}

Implementing an MIMO-OFDM communication system and comparing the system when converting the transmitted bits to residue coding (RNS-OFDM), and again when using LDPC algorithm as FEC Scheme (FEC-RNS-OFDM).

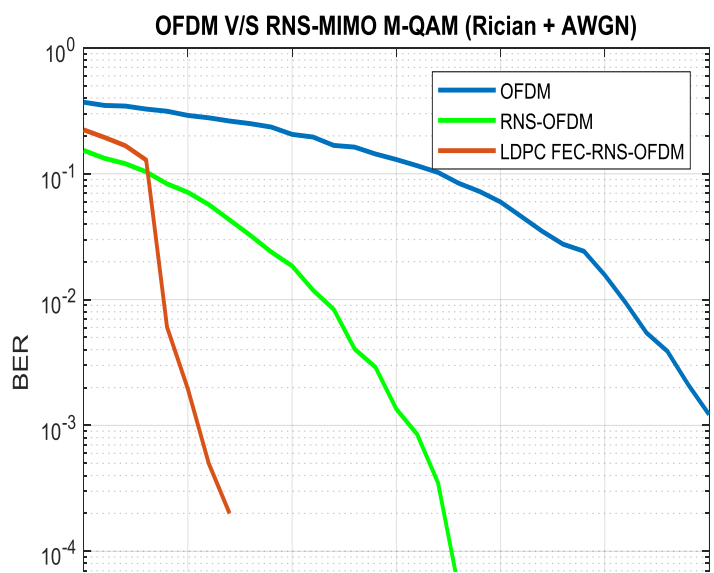

Over a Rician + AWGN channel

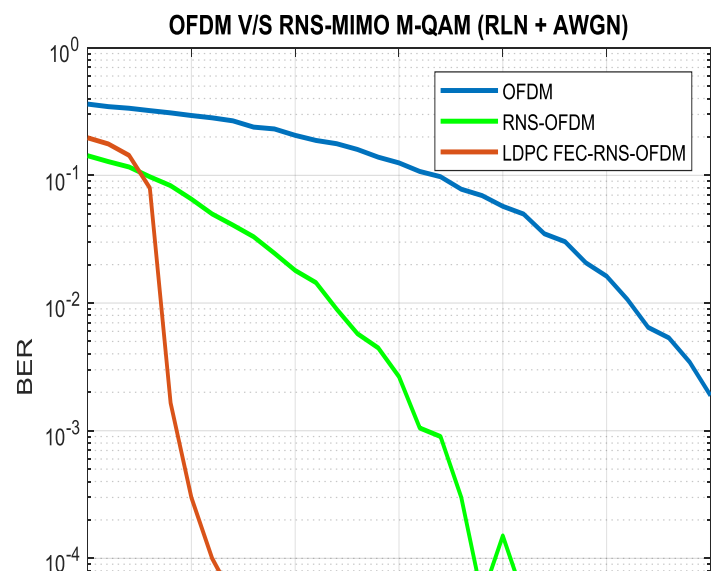

Over a RLN + AWGN channel

Figure 6. MIMO-OFDM RNS system with FEC coding schemes

Where; we could see in Figure 6 the performance enhancement done going from OFDM to RNSOFDM then implementing an FEC-LDPC scheme. Also, the effect over different channels (small and deep fading) has been analyzed.

\subsection{Comparison between different Error correction techniques}

After analyzing in previous subsections the utilization of equalizers and coding techniques as an error correction schemes; both techniques are compared over RLN + AWGN channel as seen in Figure 7. 


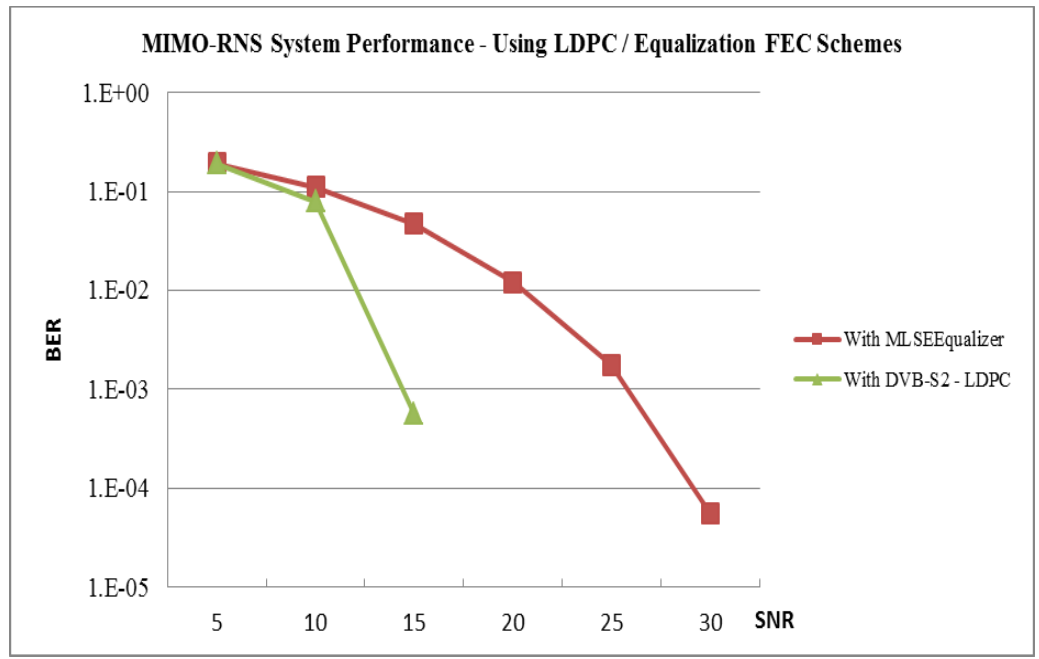

Figure 7. MIMO-OFDM RNS system with FEC schemes

In Figure 7 above, it is shown that LDPC coding provide enhanced performance over MLSE equalizer as an error correction scheme especially for higher SNR.

\subsection{BER performance for RRNS as FEC scheme in MIMO-OFDM system}

The system performance is evaluated with respect to the proposed scheme that utilize RNS coding with redundant moduli's for MIMO-OFDM system as an alternative error correction method. A redundant error correction using RNS is utilized where RNS moduli's are $\{3,5,7\}$, and redundant set are $\{11\}$.

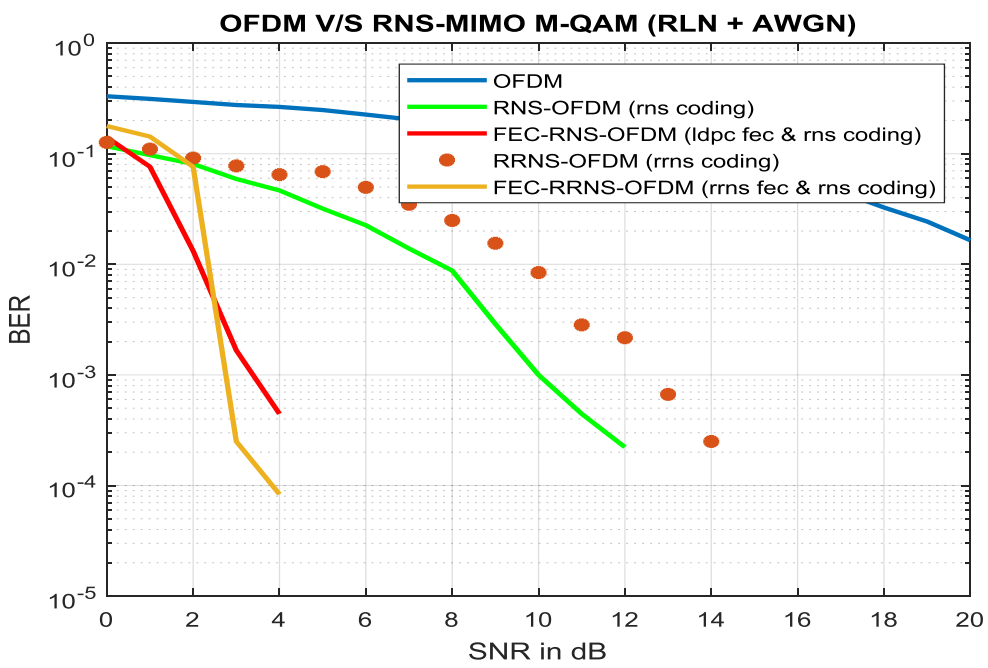

Figure 8. MIMO-OFDM system performance

From the above Figure 8, it is shown that error correction scheme with redundant RNS provide a comparable performance with that using LDPC scheme that is currently used in DVB-S2 systems.

\subsection{BER for RNS vs. RRNS schemes in MIMO-OFDM system}

Using RNS moduli's $\{3,5,7,11\}$, and redundant set initially with moduli $\{13\}$ then with redundant moduli's $\{13,17\}$, evaluating the system performance with and without redundant moduli's as seen in Figure 9. 


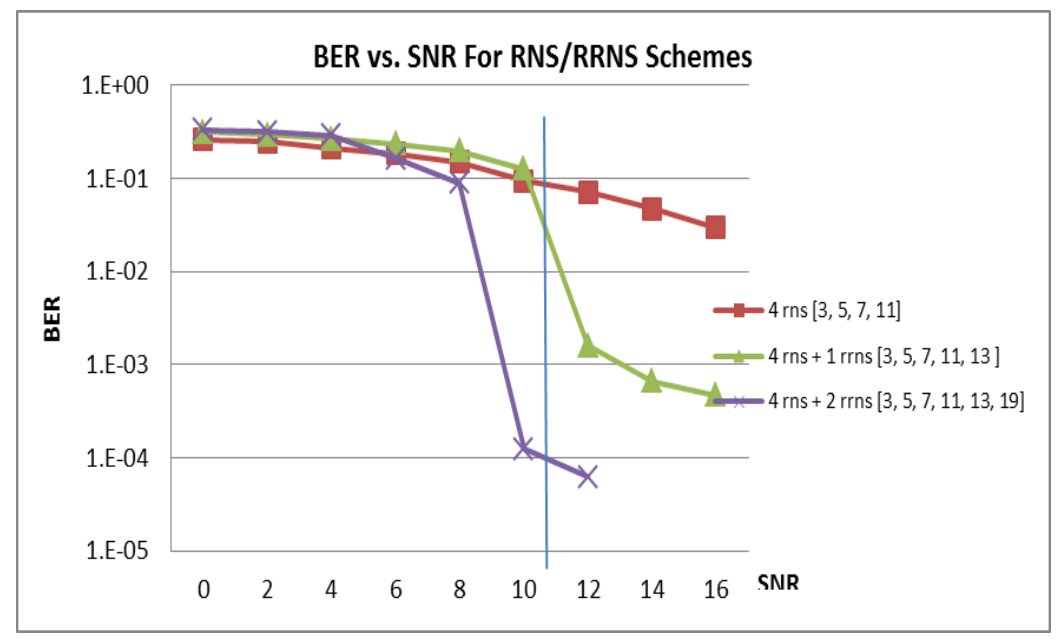

Figure 9. RRNS vs. RNS Performance comparison

From the above Figure 9, it is shown that for a SNR $=11 \mathrm{db}$, an enhancement of the BER by $1 \mathrm{~dB}$ is seen when using 1 redundant moduli, and $3 \mathrm{~dB}$ when using 2 redundant moduli's in-comparison to the system without redundant moduli's.

\subsection{PAPR performance for MIMO-OFDM system with RRNS as FEC}

For a MIMO-OFDM system over an ITU LOS + AWGN fading model channel, the PAPR for the transmitted signal is as shown in Figure 10.

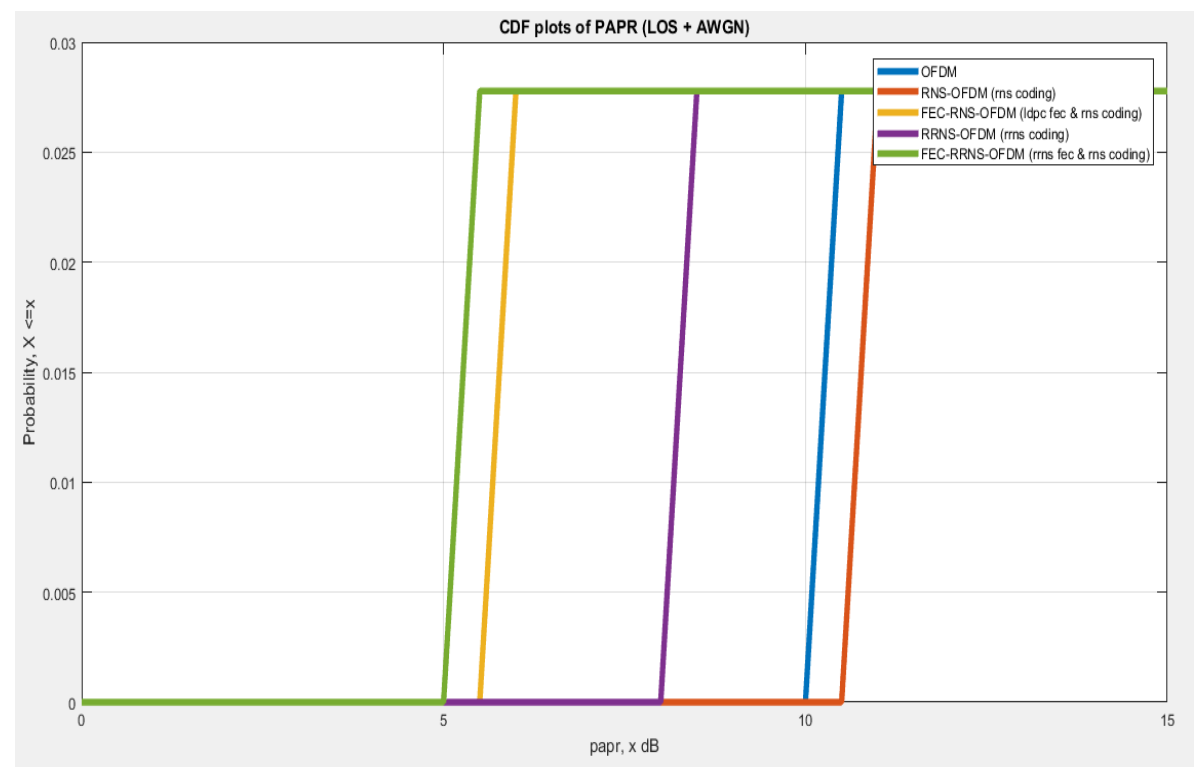

Figure 10. PAPR measurement for MIMO-OFDM system

From the above Figure 10, it is shown that the system with RRNS has the minimum signal amplitude by about $20 \%$ compared with LDPC FEC scheme (FEC-RNS-OFDM) and better than the systems that don't use FEC schemes.

\subsection{Effect of increasing RNS moduli on channel capacity} moduli sets.

In Figure 11 a MIMO-OFDM system is analyzed over a RLN + AWGN channel for various RNS 


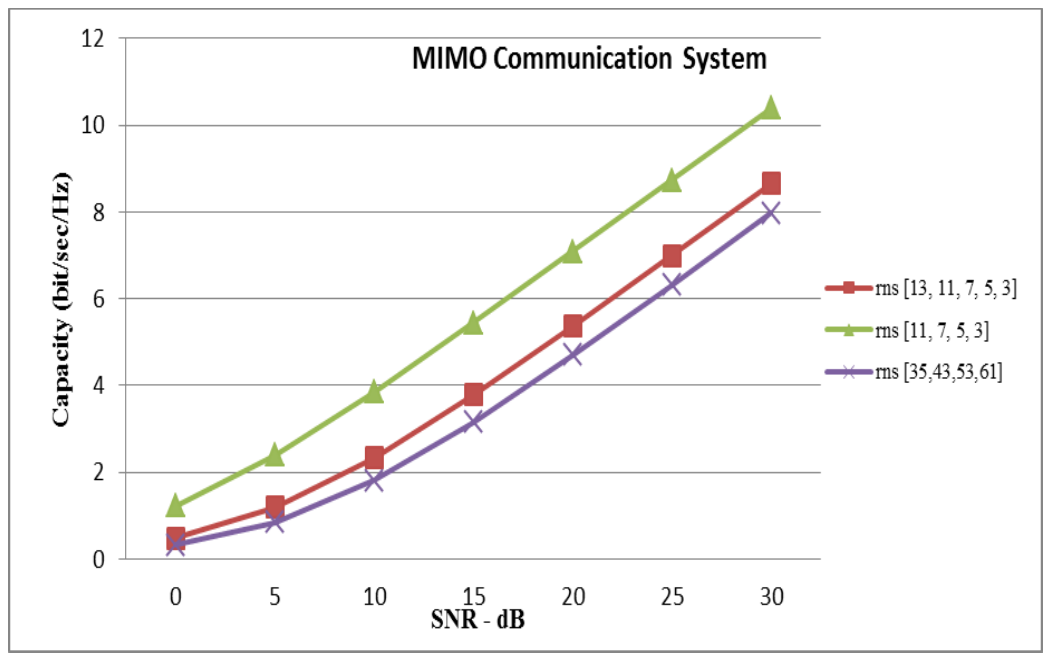

Figure 11. Channel Capacity for various RNS moduli sets

Where, we could see from Figure 11 that the increase of number of RNS moduli value leads to a decrease in channel capacity due to the increased amplitude representation, and thus it is better to select a low order moduli set to be able to enhance the system performance.

\section{CONCLUSION}

The utilization of RNS coding system with parallel distributed arithmetic and with no dependence between the different arithmetic blocks would simplify the overall design and reduces the complexity of the individual building blocks.

The paper provides an error detection and correction scheme using RRNS. Where a four and then a six-length moduli set have been proposed; in the first time three out of the four were information moduli and one was a redundant moduli, and in the second the first four moduli set is the information moduli and the last two is the redundant moduli respectively. Thus, a one and again two redundant moduli's were used for error detection and correction.

Through the performed simulations it was proven that this system provide less receiving system complexity and the straight forward error detection algorithm due to the absence of carry propagation between the arithmetic blocks, reduced dynamic power by about $20 \%$ due to the usage of small arithmetic units, and finally enhanced error detection and correction features, which improves as the redundant moduli increase taking benefit from the independent transmission feature; where an error in one sub-channel in RNS is not propagated into the other sub-channels and thus isolating the faulty residuals and as a consequence allow for fault tolerance and facilitate error detection and correction, but on the other hand this comes on the expense of reducing the available communication system channel capacity.

\section{REFERENCES}

[1] H. Bolcskei, "MIMO-OFDM Wireless Systems: Basics, Perspectives, and Challenges", in IEEE Wireless Communications, vol. 13, no. 4, pp. 31-37, Aug. 2006.

[2] A. J. Paulraj, et al., Introduction to Space-Time Wireless Communications, Cambridge, UK: Cambridge Univ. Press, 2003.

[3] Jatinder Singh and Jaget Singh, "A Comparative study of Error Detection And Correction Coding Techniques", 2012 Second International Conference on Advanced Computing \& Communication Technologies.

[4] B. Vucetic and J. Yuan, Turbo Codes: Principles and Applications. Springer Verlag, 2000.

[5] DVB-S. EN 300421 V1.1.2 (08/97), "Digital Video Broadcasting (DVB); Framing Structure, Channel Coding and Modulation for 11/12 GHz Satellite Services", ETSI, 1997.

[6] DVB-S2. EN 302307 V1.1.2 (06/06), "Digital Video Broadcasting (DVB); Second Generation Framing Structure, Channel Coding and Modulation Systems for Broadcasting, Interactive Services, News Gathering and other Broadband Satellite Applications", ETS, 2006.

[7] M. Roshanzadeh, et al., "Using Residue Number Systems for Improving QoS and Error Detection \& Correction in Wireless Sensor Networks, Communication Software and Networks (ICCSN)", May 2011 IEEE 3rd International Conference on, pp. 1-5. 
[8] K. W. Watson, "Self-checking Computations using Residue Arithmetic”, Proc. IEEE, vol. 54, pp. 1920-1931, Dec. 1966.

[9] E. D. D. Claudio, et al., "A Systolic Redundant Residue Arithmetic Error Correction Circuit", IEEE Trans. Computers, vol. 42, pp. 427-432, Apr. 1993.

[10] A. Omondi, Residue Number Systems: Theory and Implementation, Imperial College Press, 2007.

[11] Pallab Maji, Application of Residue Arithmetic in Communication and Signal Processing, Master of Science. National Institute of Technology, Rourkela, Orissa-769008, India, Juni 2011.

[12] Aingel James, et al., "Multiple error correction using non-binary Redundant Residue Number System", India Conference (INDICON) 2015, 2015, ISSN 2325-9418.

[13] Hari Krisna, et al., "A coding Theory Approach to Error Control in Redundant Residue Number Systems- Part I: Theory and Single Error Correction", IEEE Transactions on Circuits and Systems II: Analog and Digital Signal Processing, vol 39, no. 1, pp 8-17, Jan 1992.

[14] Salifu Abdul-Mumin and Kazeem Alagbe Gbolagade, “An Improved Redundant Residue Number System Based Error Detection and Correction Scheme for the Moduli Set", Advances in Wireless Communications and Networks Journal, vol. 2, no. 1, pp. 11-14, 2016.

[15] Sandeep Kaur and Gurpreet Bharti, "Orthogonal Frequency Division Multiplexing in Wireless Communication Systems: A Review”, International Journal of Advanced Research in Computer Engineering \& Technology, vol. 1, no. 3, May 2012.

[16] Vatalaro, et al., "The Generalized Rice Lognormal Channel Model - First and second Order Statistical Characterization and Simulation”, International Journal of Satellite Communications, 20, pp. 29-45, 2012.

[17] Paredes, M., "Reduction of Peak-to-Average Power Ratio in OFDM Systems", 2015.

[18] Ramoni Adeogun, "Capacity and Error Rate Analysis of MIMO Satellite Communication Systems in Fading Scenarios”, International Journal of Electrical and Computer Engineering (IJECE), vol. 4, August 2014. 